\title{
Up Close: University College London Department of Electronic and Electrical Engineering
}

\author{
lan W. Boyd \\ Editor's Note: This is the tenth article in a series focusing on the research capabilities and goals of interdisciplinary laboratories \\ pursuing materials research in universities, industry, and government
}

University College London (UCL), the largest and oldest college within the University of London, established the first chair in Electrical Technology in the United Kingdom some 101 years ago. It was here in 1904 that Ambrose Fleming patented the thermionic valve, a device that could be considered to have initiated modern electronics. The 1980s have seen one of the biggest expansions in the department's history. As a consequence of the government's shift-to-engineering scheme, student places have been expanded by $50 \%$, and the addition of 10 new members has increased the number of academic staff to 30 . Several major research awards, together with generous industrial funding from the General Electric Company have greatly assisted in expanding an evergrowing materials-based research environment. The department annually attracts a turnover of more than $£ 2 \mathrm{M}$ and contributes an average of 75 papers per year to the scientific literature.

The department is divided into three major operating groups of Physical Electronics, Systems, and Computing under the direction of Prof. J.E. Midwinter, who occupies the British Telecom Chair of Optoelectronics, Prof. D.E.N. Davies, head of the department, and Prof. J.B. Davies. Current research efforts in materials within these groups are described below.

\section{Materials for Integrated Optics}

Almost all the known methods of depositing thin optical quality dielectric, and semiconductor, films have been explored in the Thin Film Laboratory at UCL. This laboratory, under the direction of Chris Pitt, has researched a wide range of materials and process technologies for integrated optical and waveguiding acousto-optic devices. The following gives an outline of one of the ten or so current projects targeted at providing optical thin films and device structures for the field of integrated optics.

Lithium Niobate $\left(\mathrm{LiNbO}_{3}\right)$ has emerged as arguably the most important material for integrated-optics devices because it has relatively high electro-optic and piezoelectric coefficients. Thin-film optical waveguides may be fabricated in this material by several processes, including diffusion of transition metal dopants and proton exchange. Pitt's unique research on the use of molec- ular beam epitaxy for oxide film deposition has led to a novel method for the growth of $\mathrm{LiNbO}_{3}$ layers for waveguiding application on both $\mathrm{LiNbO}_{3}$ and sapphire substrates.

Figure 1 shows the equipment used at UCL. A two-chamber system with sample transfer facility enables growth and substrate/film studies to be undertaken in an optimized vacuum environment. The diagnostic chamber also serves as a load-lock, preserving a $10^{-9}$ torr vacuum while changing samples. One of the source materials, niobium, has a very low vapor pressure and requires heating to very high temperatures $\left(\sim 2400^{\circ} \mathrm{C}\right)$ so that electron beam evaporators are used as elemental sources for both the niobium and lithium beams. Gaseous oxygen is admitted via a multichannel capillary array mounted close to the substrate. Extensive cryoshielding is employed for improved vacuum in the deposition region; together with differential pumping in the substrate region, this minimizes the oxygen overpressure in the rest of the chamber. The substrate holder can be heated by electron bombardment to over $1000^{\circ} \mathrm{C}$.

This new growth method for producing epitaxial layers of lithium niobate may offer an alternative fabrication process for devices previously fabricated in $\mathrm{Ti}: \mathrm{LiNbO}_{3}$. The growth of epitaxial layers on semicon- ducting substrates is currently being investigated. The integration of electronic, optical, and acoustic devices on a single substrate may then be achievable. Current work is directed at improved control of the film crystal structure; optical, acoustic, and electro-optic characterization; and measurement of the optical-damage threshold of the film.

\section{Laser Processing of Microstructures}

One of the newer areas of thin film processing recently initiated at UCL involves the localized laser modification and growth of layered structures, an effort directed by Dr. Ian $W$. Boyd. The group studies a variety of novel photon-initiated and enhanced surface physical and chemical processes. Current funding supports investigations into enhanced silicon oxidation reactions, and nitride formation using the newly installed arc lamp and $20 \mathrm{~W}$ argon laser facilities. The highest quality laser-produced insulators reported to date have been fabricated at UCL.

Future aims include isolating and identifying the nonthermal photon-induced mechanisms introduced into the oxidation reaction. Preliminary steps have confirmed previous observations of a clear wavelength dependence to the process, as

Continued
Deposition chamber

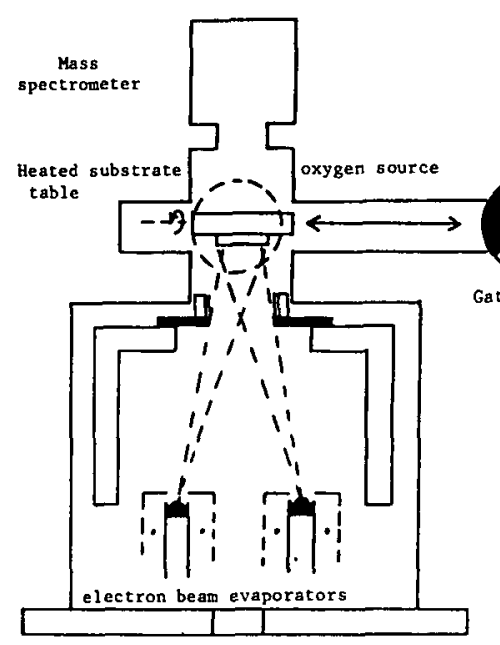

UHv pumps
Diagnostic chamber

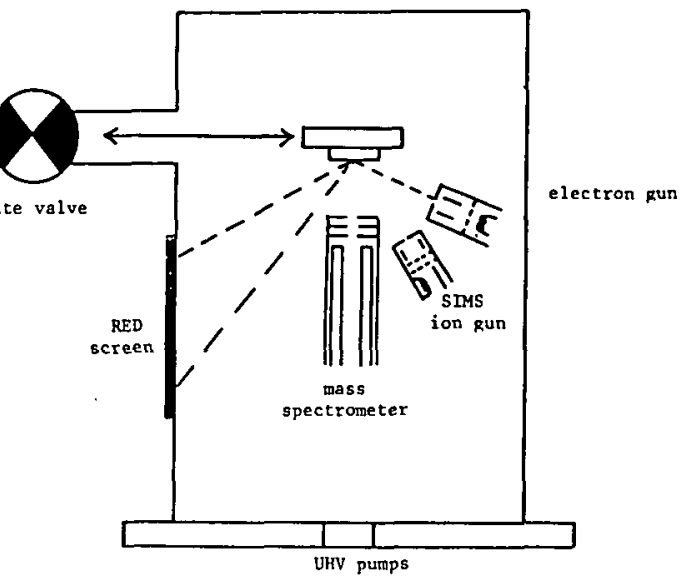




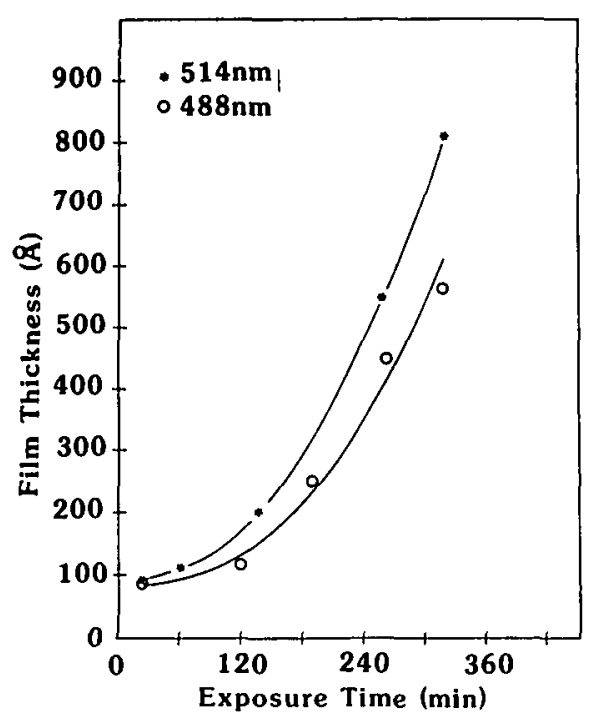

Figure 2. Experimental evidence for photon-enhanced oxidation of silicon is confirmed by the increased oxidation rate induced by longer wavelengths when equal powers of laser radiation are used.

shown in Figure 2. Additional projects, associated with laser processing linked to external industrial and governmental laboratories, include localized etching and repair of microelectronic devices, and infrared semiconducting materials, and structural modification of variously prepared metal oxide layers.

\section{Devlce Fabrication}

An industrial-standard clean room, supervised by Dr. Margaret Stallard, exists within the Department for fabricating integrated optics devices in environments to class 100 . This facility greatly enhances the progress of many other research projects demanding special geometric designs and structures. These conditions allow fine line photolithography as a standard process for integrated optic device fabrication. Devices which include three-micron line-width features are fabricated in lithium niobate using titanium in diffusion techniques. Liftoff and plasma etching techniques are available for waveguide fabrication; and following testing, metal electrodes are deposited using standard thermal deposition.

Integrated optic waveguide structures can also be fabricated by the in-house ion exchange process in glass, and by the proton exchange facility in lithium niobate.

In addition to integrated optic device fabrication, the clean room carries out plasma etching of silicon for transducer fabrication by the Systems group. Assessment of these processes is greatly assisted by scanning electron microscopy. Photolithographic and etching facilities are also employed for digital optics device fabrication in multi quantum well materials.

\section{Digital Optics}

The digital optics program started three years ago when Gareth Parry from RSRE Malvern and John Midwinter from British Telecom joined the department. The program is concerned with the development of novel optoelectronic logic devices and architectures for switching systems. The group has chosen to concentrate on $\mathrm{GaAs}$ multiple quantum well structures for most of the device development. The discovery of the quantum-confined Stark effect by AT\&T has opened up a totally new range of possibilities in the digital optics area, and the UCL group have developed a new logic element which switches with microwatts of optical power and provides hard limited outputs. The group collaborates closely with the Science and Engineering Research Council III-V facility at Sheffield, who provide samples grown by MOVPE, and with Philips Research Laboratory, who have expertise in MBE growth of quantum well structures. Only through a collaborative activity has it been possible to demonstrate novel devices in a short time scale. A new collaborative activity involving Plessey and again Sheffield University involves studies of electro-optic effects in GaInAs quantum well materials.

\section{Fiber-Optic Sensors}

The Systems group within the Department of Electronic and Electrical Engineering also has interests in materials research. One project just begun under the direction of Dr. Ian Giles will concern study of nonlinear optical effects in thin films in intimate contact with the evanescent field regions of fiber-optic couplers. Another project, directed by Dr. Shalini Venkatesh and well under way, concerns the fabrication of silicon-based microstructures and their integration into fiber-optic sensor systems. The various processes involved photolithography, reactive ion or wet chemical etching, evaporation or sputter coating, silicon-silica bonding, and examination by light or scanning electron microscopy - are all performed in-house (see Device Fabrication above), which allows systematic studies to be carried out relatively easily.

Figure 3 shows an aluminum-coated silicon dioxide micro-resonator $(200 \mu \mathrm{m}$ long, $0.5 \mu \mathrm{m}$ thick, and $5-14 \mu \mathrm{m}$ wide) in an intermediate stage of reactive ion etching. The completed device can be driven into mechanical vibration by the absorption of fractions of a microwatt of light at the metallic surface. If the frequency of the intensity modulation applied to this incident light is adjusted to match a natural mechanical resonance of the device, the basic element of a purely optical resonant sensor is achieved. This work is expected to have applications in many fields, including physiological monitoring, and is currently attracting several sponsors.

\section{Materlals Evaluation}

UCL has a long-standing and continuing interest in imaging for both nondestructive evaluation and high resolution microscopy. UCL is presently researching acoustic, scanning optical, and thermal imaging techniques. The common theme to these activities is that they all involve scanning the object relative to the source and detector. These topics were part of the plenary session at the 1986 European MRS meeting and were presented by Dr. Mike Somekh, director of the group.

Acoustic microscopy is particularly effective for subsurface imaging of opaque materials and determining the mechanical properties of materials. For imaging well below the surface in a conventional pulse echo mode, the technique is finding increasing application for defect detection in integrated circuit packages. When imaging near the surface, very high sensitivity can be achieved because a high numerical aperture acoustic lens excites Rayleigh waves on the surface, which interact with the sample to within a few wavelengths. This technique can detect delaminations, quantitatively measure bond strengths, and assess the characteristics of surface cracks. UCL is also involved with the development of signal processing techniques to improve quantitative interpretation, using frequency coding to separate topographical information from the sample reflectivity. (See Figure 4.)

Scanning optical microscopy has several advantages over conventional microscopy, since both optical and electronic processing of the signal can greatly increase sensitivity. One of our newer systems involves forming an interferometer in which the two beams are separated not in space, but by orthogonal polarization and imaging using a Nomarski objective to make a scanning Nomarski microscopy linearly Continued

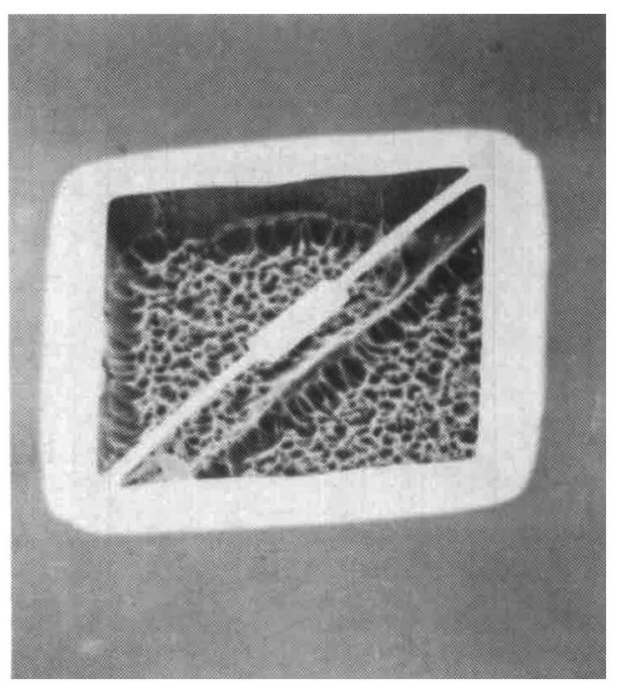

Figure 3. Al-coated lon-etched $\mathrm{SiO}_{2} \mathrm{mi}-$ cro-resonator partially reactive. 


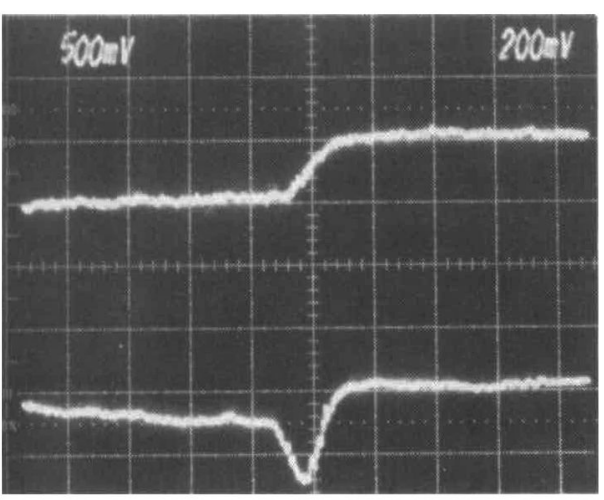

Figure 4. The lower trace shows amplitude output from a scan on a step. The dip corresponds to reduced amplitude due to nonspecular scattering. The top trace shows the pure topography signal corresponding to the average time of flight of the return signal.

sensitive to the phase shift suffered by the two beams. A conventional system is sensitive only to the square of the phase difference between the signals. This linear sensitivity greatly improves sensitivity to minute topography: less than $10 \AA$ can be detected in a $1 \mathrm{~Hz}$ bandwidth. The fact that both beams have a common path greatly reduces the effects of microphonics, since both interfering beams suffer similar path perturbations.

UCL is also working on a system which may enable distinguishing between phases of the reflected signal and true topography. High sensitivity to small lateral features makes the microscope particularly useful for examining small features, such as stacking faults on semiconductor substrates, prior to processing.

UCL is involved in two implementations of thermal microscopy. The first heats the sample periodically with a bias portmodulated semiconductor laser, expanding the sample surface. The expansion is detected interferometrically with a heterodyne interferometer focused on the same spot as the heating beam. This system has been used to monitor ion implantation doses in semiconductors, proving extremely sensitive to very small amounts of residual damage, even in flash-annealed samples.

The second instrument has been used for studying electromigration and for subsurface imaging. It heats the sample in the same way as the interferometric system, but the effect of the sample heating is detected by the variation of the emitted blackbody radiation at the chopping frequency. The black-body radiation is separated from the reflection by a germanium beam splitter so that the microscope is capable of taking thermal and optical images simultaneously. We are presently comparing the instrument with the Rayleigh wave mode of the acoustic microscope for characterizing thin films. The instrument also seems to have applications in semiconductor characterization, since the heating laser can excite carriers which perturb the emissivity directly, thus giving a signal dependent on electrical rather than thermal properties of the material. It has been observed that semiconductors with long carrier lifetime give much a larger signal than those with short carrier lifetime, which provides a strong contrast mechanism of semiconductor surface. This technique is being developed to establish a noncontacting high resolution means of measuring carrier lifetimes and mobility.

The Electronics and Electrical Engineering Department at UCL has traditionally maintained close collaborations with industry. The level of external support for our research now exceeds the total internal running costs of teaching 250 bachelor's degree students and 75 postgraduate master's degree students, and training 65 students performing $\mathrm{PhD}$ research. Establishment of the British Telecom Chair of Optoelectronics and three other industrial faculty positions at UCL, and the achievement of an outstanding rating by the University Grants Committee, further enhance the ever-growing international reputation of England's third oldest university.

Ian W. Boyd obtained his BSc and PhD in applied physics at Heriot-Watt University, Scotland. After two years as a visiting scientist at the Center for Applied Quantum Electronics at North Texas State University, he joined UCL's Electronic and Electrical Engineering Department in 1984. His studies include laser processing, nonlinear optical phenomena, and the oxidation of silicon, in which he is also an industrial consultant.

University College London

Torrington Place

London WC1E 7JE

Telephone 387-7050, Ext. 3956

\section{AAAS/WESTINGHOUSE AWARD}

FOR

\section{Public Understanding OF Science AND Technology}

\section{NOMINATIONS INVITED}

- New annual Award for working scientists and engineers from all disciplines who make outstanding contributions to public understanding of science and technology and are not members of the media.

- First Award will be presented during the AAAS Annual Meeting in Boston, February 11-15, 1988.

- Award carries a $\$ 2,500$ prize.

- Sponsored by Westinghouse Electric Fund and American Association for the Advancement of Science.

Contact: Patricia S. Curlin, AAAS Committee on Public Understanding of Science and Technology, $1333 \mathrm{H}$ Street N.W., Washington, DC 20005. Telephone (202) 326-6600. 